\title{
Antiviral treatment normalizes neurophysiological but not movement abnormalities in simian immunodeficiency virus-infected monkeys
}

\author{
Howard S. Fox, ${ }^{1}$ Michael R. Weed, ${ }^{1}$ Salvador Huitron-Resendiz, ${ }^{1}$ Jamal Baig, ${ }^{1}$ \\ Thomas F.W. Horn, ${ }^{1}$ Peter J. Dailey, ${ }^{2}$ Norbert Bischofberger, ${ }^{3}$ and Steven J. Henriksen ${ }^{1}$ \\ ${ }^{1}$ Department of Neuropharmacology, The Scripps Research Institute, La Jolla, California, USA \\ ${ }^{2}$ Bayer Diagnostics, Nucleic Acid Diagnostics, Emeryville, California, USA \\ ${ }^{3}$ Gilead Sciences, Foster City, California, USA \\ Address correspondence to: Howard S. Fox, Department of Neuropharmacology, CVN-8, The Scripps Research Institute, \\ 10550 North Torrey Pines Road, La Jolla, California 92037, USA. Phone: (858) 784-7171; Fax: (858) 784-7296; \\ E-mail: hsfox@scripps.edu. \\ Received for publication December 9, 1999, and accepted in revised form May 30, 2000.
}

\begin{abstract}
Simian immunodeficiency virus (SIV) infection of rhesus monkeys provides an excellent model of the central nervous system (CNS) consequences of HIV infection. To discern the relationship between viral load and abnormalities induced in the CNS by the virus, we infected animals with SIV and later instituted antiviral treatment to lower peripheral viral load. Measurement of sensoryevoked potentials, assessing CNS neuronal circuitry, revealed delayed latencies after infection that could be reversed by lowering viral load. Cessation of treatment led to the reappearance of these abnormalities. In contrast, the decline in general motor activity induced by SIV infection was unaffected by antiviral treatment. An acute increase in the level of the chemokine monocyte chemoattractant protein-1 (MCP-1) was found in the cerebrospinal fluid (CSF) relative to plasma in the infected animals at the peak of acute viremia, likely contributing to an early influx of immune cells into the CNS. Examination of the brains of the infected animals after return of the electrophysiological abnormalities revealed diverse viral and inflammatory findings. Although some of the physiological abnormalities resulting from SIV infection can be at least temporarily reversed by lowering viral load, the viral-host interactions initiated by infection may result in long-lasting changes in CNS-mediated functions.
\end{abstract}

J. Clin. Invest. 106:37-45 (2000).

\section{Introduction}

HIV-1-associated cognitive/motor disorder (also known as neuroAIDS) occurs in approximately one third of individuals infected with HIV $(1,2)$. The dramatic lowering of viral load with highly active antiretroviral therapy (HAART) has had an important impact on HIV1-associated dementia (a severe form of neuroAIDS), the incidence of which has declined by approximately onehalf $(3,4)$. In contrast to the lowering of the incidence, the effects of HAART on the prevalence of HIV-1-associated dementia and on HIV-induced minor cognitive and motor disorders remain uncertain; conceivably, the prevalence may even increase, given that people with HIV infection are living longer.

Ominously, recent epidemiological data document not only an almost $50 \%$ increase in AIDS dementia complex (ADC) as an AIDS-defining illness after the advent of HAART, but also a greater than twofold increase in the median CD4 count at time of ADC diagnosis since the introduction of HAART (5). In part, such results may be due to the finding that most antiviral agents do not efficiently penetrate the blood-brain barrier (BBB) or are actively transported out of the central nervous system (CNS) (6). Even after antiviral treat- ment that successfully controls virus in the immune compartment, the CNS may suffer continuing damage induced by HIV infection. Furthermore, the unavailability of these drugs to many patients, the failure of some drug regimens to control virus in numerous infected individuals, and the potential for development of resistant viruses with chronic use of these agents also put the CNS at risk from damage by HIV.

SIV infection of rhesus monkeys provides an excellent model of HIV infection and AIDS, including the CNS sequelae (7-9). Within the first 3 months after SIV inoculation of monkeys, we found that measurement of sensory-evoked potentials revealed infection-induced abnormalities in the CNS neuronal circuitry (10). Such electrophysiological analysis of evoked potentials, which measures the electrical activity produced by groups of neurons after stimulation, also uncovers abnormalities stemming from HIV infection. Sensory-evoked potentials, e.g., responses to such stimulation as sound or light, and event-related potentials, e.g., recognition of a rare event in the stimulation pattern, can be altered in both asymptomatic and symptomatic HIV-infected individuals (11-14). Additionally, we and others have demonstrated motor abnormalities in SIV-infected monkeys (10, 
15-17). Motor abnormalities are an integral part of the HIV-1-associated cognitive/motor disorder, and psychomotor slowing is a predictor of developing AIDS, AIDS dementia, and shortened survival (18).

Although some prophylactic and symptomatic therapeutic trials are in progress, the lowering of peripheral viral load with antiretroviral drugs remains the key mode of treating HIV-induced CNS complications. Yet the relationship of viral load to CNS dysfunction remains unknown. To analyze such relationships, we used the acyclic nucleoside phosphonate (nucleotide) analog 9-(2-phosphonylmethoxypropyl)-adenine (PMPA; a reverse transcriptase inhibitor) to reduce plasma viral load in SIV-infected monkeys $(19,20)$. Although this treatment effectively reversed the virally induced electrophysiological abnormalities, no effect was found on fever or movement. Presumably, after a period of the initial viremia and CNS infection, an independent cascade of events unleashed long-lasting changes in CNS-mediated functions.

\section{Methods}

Animals. Rhesus monkeys, free from SIV, type D simian retroviruses, and herpes B virus, were obtained from Charles River Breeding Laboratories (Key Lois, Florida, USA). All animal experiments were performed with approval from The Scripps Research Institute Institutional Animal Care and Use Committee. Animals were kept in a containment facility and handled for experiments after intramuscular administration of anesthesia with ketamine. Blood samples were drawn from the femoral vein, and cerebrospinal fluid (CSF) was obtained from the cisterna magna. For surgical implantation of the telemetry transmitters, each animal received general anesthesia. The transmitter was implanted aseptically into a subcutaneous pocket dissected in the right flank. Necropsy was performed after terminal anesthesia. Animals were perfused with sterile PBS containing $1 \mathrm{U} / \mathrm{mL}$ heparin before samples were taken for SIV quantification and for formalin fixation and paraffin embedding.

Viral infection and detection. SIV inoculation was performed via the saphenous vein using a cell-free viral stock (SIVmac182) obtained after serial passage of SIVinfected microglia (10). Animals received $1 \mathrm{~mL}$ of SIVmac182 in RPMI-1640, containing $0.5 \mathrm{ng} / \mathrm{mL}$ p27 (gag) antigen; this dose is equivalent to $10^{3}$ tissue culture infectious doses when titered on CEMx174 cells. To measure plasma viral load, plasma was separated from cells in EDTA-anticoagulated blood by centrifugation. Plasma and CSF SIV RNA viral load was measured using the quantitative branched DNA (bDNA) signal amplification assay, performed at Bayer Reference Testing Laboratory (Emeryville, California, USA). For these fluids, viral particles were pelleted by centrifugation from measured volumes and lysed, and the bDNA signal amplification assay was performed using probes that hybridize within the pol region of SIV as described previously (21). Viral loads in tissue samples were also determined by the bDNA assay in a manner similar to that developed for liver hepatitis $\mathrm{C}$ quantification (22) and adapted for SIV quantitation in tissues (23). Samples from each region were measured in duplicate, and the average value was reported.

Virus recovery from the CSF was performed by a coculture assay. Cells were separated from the CSF by centrifugation, and the presence of cell-associated virus was identified by adding cells to cultures of CEMx174 cells. The effect of productive virus infection was monitored by observation for syncytia formation; all cultures were maintained for at least 3 weeks, and the culture supernatants were assayed for production of the SIV p27 antigen by ELISA using the SIV Core Antigen kit (Beckman Coulter, Fullerton, California, USA).

Antiviral treatment. PMPA was obtained from Gilead Sciences Inc. (Foster City, California, USA) dissolved in pyrogen-free water, adjusted to $\mathrm{pH} 7.0$ with $0.1 \mathrm{~N} \mathrm{NaOH}$, diluted to $30 \mathrm{mg} / \mathrm{mL}$, and filter sterilized. Before the initiation of PMPA treatment, animals were given one daily intramuscular injection of sterile saline for 2 weeks in order to acclimate the animals to the injection protocol and to examine any effects of the injection itself. For active treatment, the saline injections were replaced by one daily injection of PMPA (at $30 \mathrm{mg} / \mathrm{kg}$ ). After the treatment period, saline injections were reinstituted for 2 additional weeks. Levels of PMPA in heparinized plasma and CSF were determined by a validated reversephase ion-pair HPLC analysis with fluorescence derivatization (24) by an independent reference laboratory (Oread Laboratories, Lawrence, Kansas, USA).

Albumin and monocyte chemoattractant protein-1 assessment. Plasma and CSF levels of albumin were determined by radial immunodiffusion (The Binding Site, San Diego, California, USA). Samples in which the CSF red blood cell count exceeded 10,000 red blood cells per microliter were excluded from analysis owing to blood contamination. The albumin quotient (CSF albumin concentration/plasma albumin concentration) was calculated. In uninfected animals, the albumin quotient was found to be less than 0.005 . Plasma and CSF concentrations of monocyte chemoattractant protein-1 (MCP-1) were determined by ELISA (R\&D Systems Inc., Minneapolis, Minnesota, USA). CSF was only available from three of the infected monkeys at the 77-day postinoculation time point, and two monkeys for the 105- and 133-day postinoculation time points.

Pathological analysis. Histopathological analysis included examination of hematoxylin and eosin-stained sections from the brain and all organs. Apart from the findings in the brain described here, examination of the rest of the animal revealed typical findings of SIV infection within lymphoid tissue, including lymphoid follicular with large, irregular, depleted germinal centers with thin mantle zones. There was no evidence of opportunistic infection or malignancy. Additional studies included immunohistochemical and in situ hybridization analysis. Immunohistochemical staining was performed with 
the antibodies LN-3 for HLA-DR, 2B11 and PD7/26 for leucocyte common antigen (LCA), Mac387 for the calprotectin antigen in activated macrophages (all from DAKO Corp., Carpinteria, California, USA), and GrB-7 for Granzyme B (Monosan, Uden, The Netherlands). Pretreatment $\left(95^{\circ}\right.$ heat in citrate buffer for LN-3, LCA and GrB-7; trypsin at $37^{\circ}$ for Mac387) was performed. Endogenous peroxidase activity and nonspecific binding were blocked. All sections were examined and scored in a blinded fashion. Controls included omission of the primary antibody and use of irrelevant primary antibodies. In situ hybridization was performed as described elsewhere (25) using [ $\left.{ }^{35} \mathrm{~S}\right]$-labeled single-strand RNA probes consisting of sense and antisense 865-bp fragments of the SIVmac nef gene, which hybridized to both spliced and unspliced SIV RNA. Experiments were performed in duplicate, counterstained with hematoxylin and eosin, coverslipped, and examined.

Electrophysiology. Electrophysiological analysis of brainstem auditory-evoked potentials (BSAEPs) was performed on ketamine $(20 \mathrm{mg} / \mathrm{kg})$ anesthetized animals as described previously $(10,26)$. Evoked events were analyzed offline using signal averaging capabilities of a microcomputer. Average peak latencies were calculated and compared between groups. A repeated measures ANOVA, with post hoc $t$ test, was used to test for statis- tical significance; significance was set at $P<0.05$.

Radiotelemetry. Continuous movement and temperature analysis was performed using radiotelemetry with implantable transmitters, cage-mounted receivers, and software data acquisition program purchased from Data Sciences (St. Paul, Minnesota, USA) as described previously (10). To account for individual differences in gross motor activity, data from individual monkeys were normalized against the average of a 14-day baseline period (preinoculation for SIV groups, preceding saline injection for the PMPA-only group). Data were then expressed as a percentage of this baseline. Daily mean temperature was determined by averaging across all such samples in a given day. Temperature data were transformed into a change in temperature from baseline by subtracting the baseline mean from each daily mean temperature. Such normalization precluded using baseline time points in an ANOVA; therefore, 95\% confidence intervals of baseline periods and treatment periods were compared. Differences were considered statistically significant if the $95 \%$ confidence intervals did not overlap. To determine the effect of PMPA treatment, a repeated-measures ANOVA was used for comparisons between the saline 1, PMPA, and saline 2 treatments. The ANOVA used the between factor of infection and the within factor of treatment (three levels: saline 1 ,

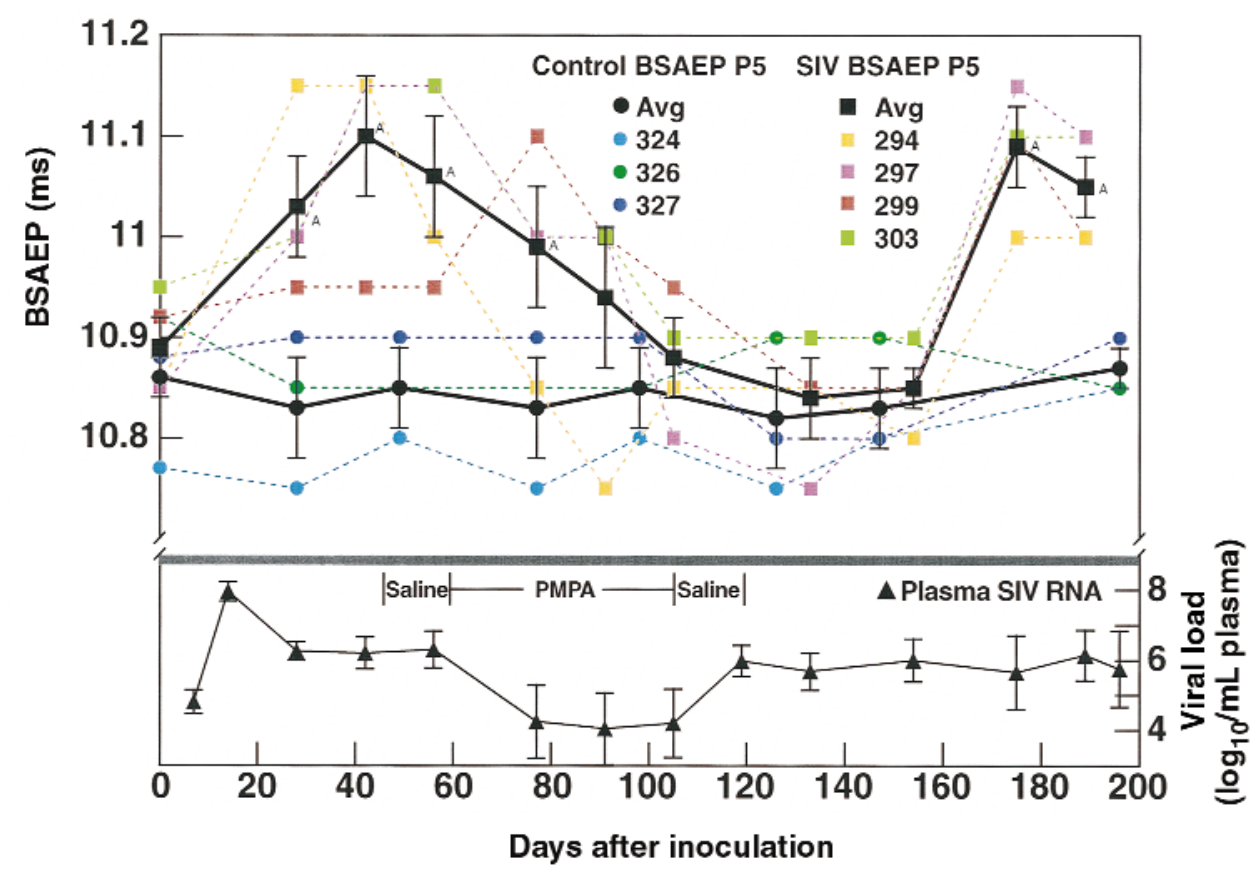

Figure 1

Effects of PMPA on SIV-induced evoked potential abnormalities. Top: BSAEP P5 latencies in groups of four SIV-infected and three uninfected control monkeys. The latency is indicated on the $y$-axis. SIV-infected animals are indicated by squares, control uninfected animals by circles. Black symbols are the group averages with error bars denoting SEM; colored symbols show data points for the individual animals. After infection, the evoked potential latency is significantly delayed 1-2 months after infection but becomes indistinguishable from preinoculation values after 1 month of PMPA treatment. When treatment is withdrawn, BSAEP latency values remain normal for approximately 2 months, then again become significantly delayed. ${ }^{A} T$ he point differs from baseline. Bottom: Copy equivalents of SIV RNA in the plasma of the infected monkeys. Plasma viral load, indicated on the $y$-axis ( $\log _{10}$ transformed, error bars indicate SEM), peaks at 14 days after inoculation, then reaches a steady-state level. Upon institution of PMPA therapy, viral load drops more than 100 -fold but recovers to pretreatment levels upon cessation of therapy. 
Figure 2

Effects of PMPA and SIV infection on movement and temperature. (a) Gross motor activity. Gross motor activity was normalized for each animal to its baseline, and data for each group were expressed as a percentage of baseline along the $y$-axis. The heights of the boxes indicate the group mean, and the error bars the SEM. Three time periods are delineated: saline injection before PMPA treatment (Saline 1); during PMPA treatment (PMPA); and saline injection after PMPA treatment (Saline 2). In the left panel, data from the SIVinfected (filled boxes) and uninfected (open boxes) animals in this study are shown. A decrease in gross motor activity followed SIV infection regardless of PMPA administration. In the right panel, data are included for comparison from the same period after viral inoculation (indicated in italics) in a previously studied untreated SIVinfected group (10). AThe point differed from baseline condition. BThe groups differed during this condition. (b) Fine motor coordination in the bimanual motor task. The latency to retrieve all 15 raisins is indicated on the $y$-axis (error bars represent the SEM) for the time points indicated as in a. Despite large changes in gross motor activity, there were no effects of either PMPA or SIV infection on fine motor control in the bimanual motor task. (c) Body temperature. Change in temperature $(\Delta T)$ from baseline, plotted on the $y$-axis with error bars indicating the SEM, was determined by subtracting the baseline mean from each daily mean temperature. The periods analyzed and the animal groups are as in a. An increase in body temperature followed SIV infection regardless of PMPA administration. In the uninfected animals, the injections resulted in a slight increase in body temperature, which was significantly above baseline during the PMPA treatment (left panel). AThe point differs from the baseline condition.

PMPA, and saline 2). Significant main effects were further evaluated using post hoc comparisons with the Tukey-Kramer procedure. Statistical analysis was performed using GB-Stat software (version 6.0; Dynamic Microsystems, Inc., Greenwich, Connecticut, USA).

Bimanual motor task testing. The bimanual motor test involved the retrieval of raisins from a plastic holeboard filled with raisins and mounted perpendicular to the front of the home cage, entailing bimanual dexterity as described elsewhere (27). The time elapsed to retrieve all 15 raisins (latency) was recorded by the experimenter with a stopwatch. Animals were trained for asymptotic performance preceding experimental manipulations. To determine the effect of PMPA treatment, a repeated-measures ANOVA was used for comparisons among results recorded in the 2 weeks before treatment (baseline), the first 2 weeks of saline administration before PMPA treatment (saline 1), the final 2 weeks of PMPA treatment (PMPA) and the 2 weeks of saline treatment after PMPA administration (saline 2). The ANOVA used the between factor of infection and the within factor of treatment (four levels: baseline, saline 1, PMPA, and saline 2).

\section{Results}

To discern the relationship of peripheral viral load to abnormalities in CNS function, we inoculated four rhesus monkeys with SIV and performed a longitudinal a

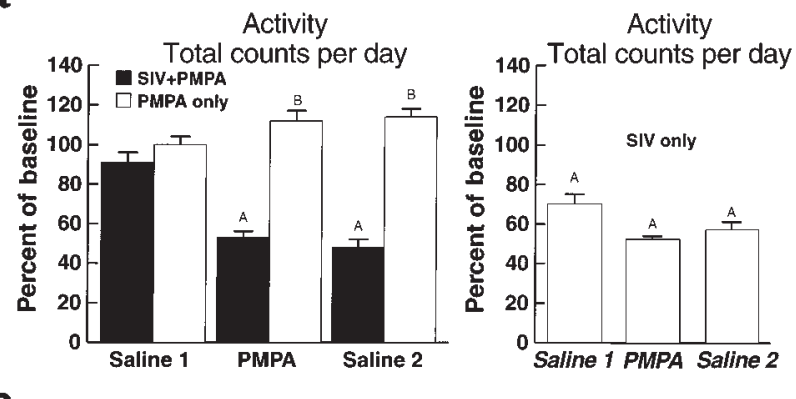

b

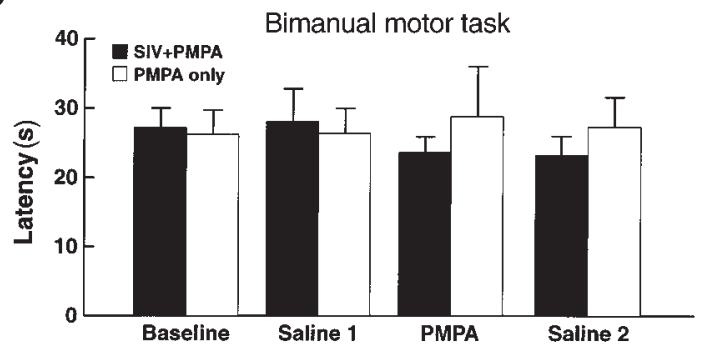

C

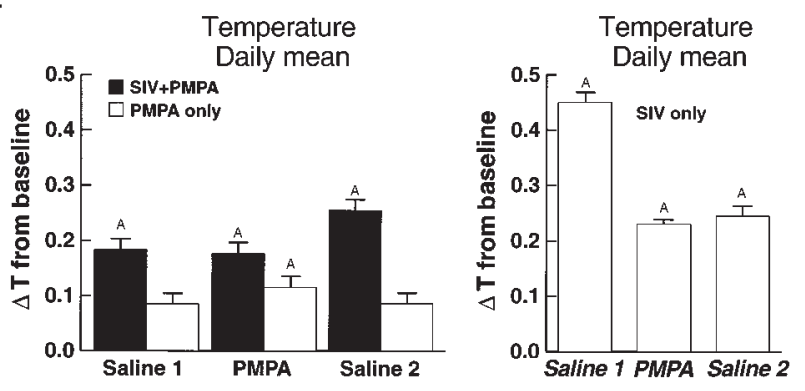

analysis of evoked potentials, movement, and temperature in comparison to three uninfected control animals. As described previously (10), the evoked potentials became abnormal early, within the first month after inoculation in the SIV-inoculated group, as illustrated by their BSAEP P5 latency values (Figure 1, top). At 2 months after inoculation, we instituted antiviral therapy with PMPA, resulting in a rapid drop in plasma viral load (Figure 1, bottom). Once the viral load decreased, the BSAEP latency normalized. PMPA therapy then continued for almost 2 months, but upon its discontinuation, the viral load increased, and with an approximately 2-month lag, the BSAEP P5 latency again became significantly delayed (Figure 1, top). PMPA therapy itself had no effect on the evoked potentials of uninfected monkeys (Figure 1, top). Viral recovery from the CSF was also affected by PMPA therapy and correlated with evoked potential delays. Before treatment, SIV could be recovered by culture from the CSF of all four infected animals at 28 days after inoculation, and three of the four at 56 days after inoculation. At the end of the treatment course, at 105 days after inoculation, no virus could be recovered from the CSF of any infected animal, and similarly no virus could be recovered 4 weeks later at 133 days after inoculation. When the evoked potentials were again delayed, at 175 days after inoculation, virus could be 
recovered from the CSF of two of the animals; at necropsy 3 weeks later, SIV could be detected in the CSF of three of the four animals.

Next, we examined the effect of viral load on motor behavior by using two tests: general motor activity (the spontaneous movement of monkeys during the day and night) and performance of a bimanual motor task (timed efficiency in extracting raisins from a holeboard). Previously we found that 3 months after SIV inoculation, general motor activity decreased by approximately 50\% (10). However, now, unlike the normalizing effect of PMPA on evoked potential, this drug had no effect on the SIV-induced decline in movement, despite the reduction of viral load (Figure 2a). Additionally, PMPA alone had no effect on gross motor activity in the uninfected controls.

A bimanual motor task was used to measure manual dexterity, procedural learning, and motivation to work for a preferred food reinforcer (raisins). This test is useful to assess the behavioral deficits induced by SIV infection in the later stages of disease $(8,16,26)$ and can serve as a control for generalized motor abnormalities or motor side effects of the treatment. In the early stages of infection, neither SIV infection nor PMPA administration had significant effects on the fine motor coordination measured during the bimanual motor task (Figure 2b). Thus, at the time of large decreases in gross motor activity, no change occurred in fine motor coordination. This suggests that the decreased gross motor activity after SIV infection, which was insensitive to PMPA treatment, did not result from a global motor deficit.

After resolution of the acute phase of SIV infection, we have found a persistent elevation of body temperature in SIV-infected monkeys (10). Analysis of the animals' average daily temperatures between baseline and treatment conditions confirmed an increase in body temperature after SIV infection regardless of PMPA administration (Figure 2c).

Because BBB leakage can occur sporadically over the course of SIV infection $(10,28)$, the BBB was monitored longitudinally by assessing the albumin quotient in the CSF. The albumin quotient (ratio of the albumin concentration in the CSF to that in plasma) remained within the normal range (less than 0.005) throughout the course of infection except for a slight elevation (of 0.006 ) in one monkey at 77 days after inoculation, during PMPA treatment. Although PMPA has not been directly studied, a closely related drug, 9-(2-phosphonylmethoxyethyl)-adenine (PMEA) is poorly accessible to the CNS after peripheral administration (29). Similarly, when measured by HPLC, PMPA could not be detected in the CSF of two SIV-infected or two control monkeys studied here during the period of therapy.

Recruitment of immune cells to the CNS involves many steps, including the production of chemoattractant molecules. Here, measurement of MCP-1 levels in the plasma and CSF of SIV-infected animals revealed a distinct elevation of MCP-1 in the CSF relative to the plasma at 14 days after inoculation (Figure 3 ) coincident with the peak of viremia, possibly contributing to the initial influx of macrophages into the brain 2 weeks after infection (30). Each infected animal exhibited increased CSF MCP-1, whereas control animals exhibited stable MCP-1 levels throughout the experiment.

The infected animals in this study were necropsied at 6 months after infection, 2 months after the termination of therapy. At that point, other than the features already described here, the animals were asymptomatic with respect to SIV infection. Infiltrating the brains were varying numbers of mononuclear cells consisting of macrophages and lymphocytes. These ranged from occasional foci of perivascular inflammatory cell clusters to more frequent but scattered perivascular and parenchymal cellular infiltrates (Table 1; Figure 4, a-c). MHC class II expression was present on the infiltrating inflammatory cells, consistent with their activation. Additionally, MHC class II expression was observed on microglia focally within the cortex and brainstem independent of the presence of other pathology (Figure 4d). Monkey 297 had the most severe pathological manifestations, consisting of a moderate degree of lymphocytic and macrophage infiltrates and rare multinulceated giant cells. This animal also had the highest plasma and CSF viral load. Virus was present in all seven regions of the brain sampled by the bDNA assay (Table 1 ), and SIV could also be detected by in situ hybridization (Figure 4e). Two other animals (nos. 294 and 299) with similar plasma viral loads had mixed CNS findings. In monkey 294, a mild degree of immune infiltra-

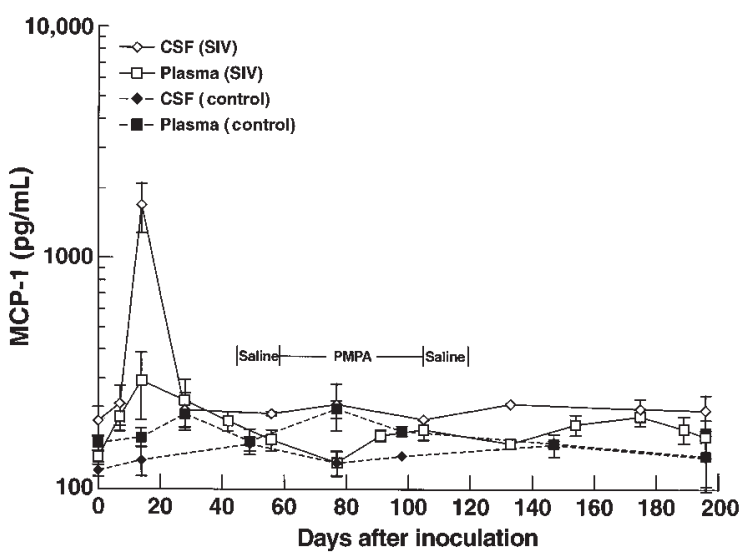

Figure 3

MCP-1 concentrations in the plasma and CSF of SIV-infected monkeys. The $y$-axis indicates the mean MCP-1 concentration in the CSF (open diamonds) and plasma (open squares) in the SIV-infected animals, and in the CSF (filled diamonds) and plasma (filled squares) over the time course of the experiment. The error bars indicate the SEM. An increased ratio of MCP-1 in the CSF relative to plasma (5.8fold) was seen at 14 days after inoculation in the infected animals, coincident with the peak of viral load. All four infected animals exhibited higher levels of MCP-1 in the CSF than in plasma at this time point. At other time points, CSF levels ranged from 0.9 to 1.8 of those in plasma. 

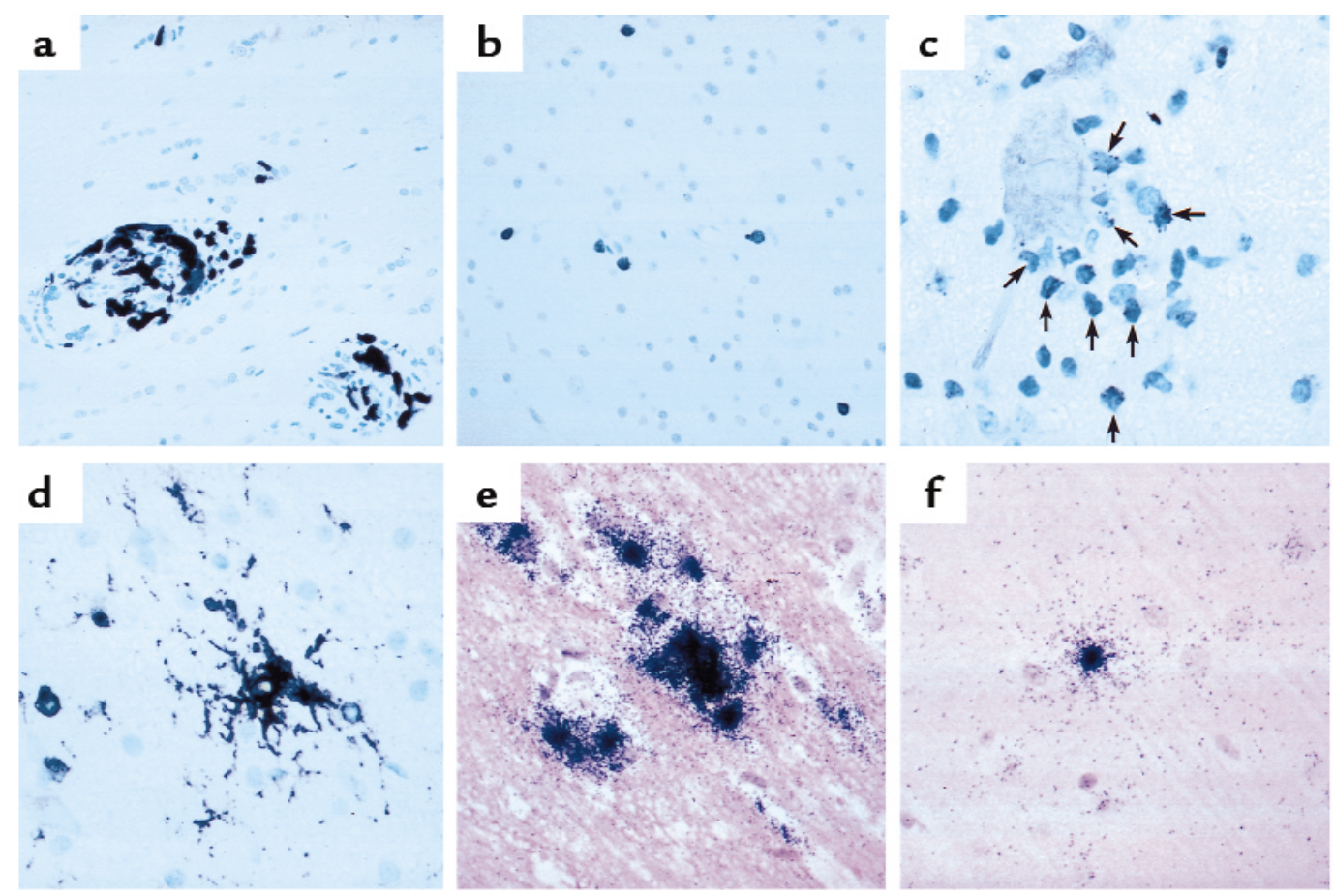

\section{Figure 4}

Histopathology in the CNS of PMPA-treated, SIV-infected monkeys. (a) Mac387 reactivity in perivascular macrophages in monkey 297. (b) LCA reactivity of infiltrating lymphocytes in monkey 294. (c) Granzyme B reactivity in infiltrating lymphocytes in monkey 303. Cells (arrows) expressing immunoreactive granules surround a neuron. (d) LN3-reactivity for HLA-DR in a cell with the morphological characteristics of a resident microglial cell (center). (e) In situ hybridization revealing cells producing SIV RNA in animal 297. (f) In situ hybridization revealing a cell producing SIV RNA in animal 294.

tion was present in the brain. Although no viral RNA was evident in the CNS by bDNA testing, occasional viral RNA-positive cells were found in the cortex as determined by in situ hybridization (Figure 4f). In monkey 299, only rare immune infiltrates could be found in the brain. Yet bDNA testing revealed virus in three of seven regions (inferior colliculus, head of the caudate, and the temporal gyrus), and SIV-positive cells were detectable by in situ hybridization. Interestingly, the CSF of monkey 299 contained almost one log less of virus than did the CSF of monkey 294, despite the finding of quantifiable SIV in the brain of monkey 299 but not in monkey 294. The fourth animal (no. 303) had only focal, but rather severe, immune infiltrates in the brain. In this animal, virus was detectable only in the spleen by bDNA; none was apparent in the plasma, CSF, or CNS. In situ hybridization also did not reveal SIVpositive cells in the CNS. Neither the severity of histopathology nor presence of virus correlated with the severity of functional abnormalities, although the small number of subjects likely precluded such a finding.

\section{Discussion}

During the early stage of SIV-induced disease tested here, the process that produced abnormalities in evoked potentials was reversible and, in fact, responded to treatment that lowered the viral load. In contrast, events leading to movement decrease and body temperature increase were unaffected by this treatment. The drug used for treatment, PMPA, probably affected the virus directly only in the periphery, not within the CNS, because of its inability to cross the BBB effectively. Still, such treatment can be effective in preventing certain untoward effects of infection on the CNS, as virally infected cells from the periphery can enter the CNS, and peripherally produced host defense molecules can affect the CNS. Once viral load is lowered in the periphery, a normal turnover of virally infected cells in the CNS will lead to decreased virus in the CNS. It is possible that the early seeding of the CNS by virus and/or the early influx of activated macrophages and other immune cells into the CNS result in changes that are unaffected by future drug treatment. Alternatively, the consequences of the continued viral-host interaction may inevitably lead to the movement and temperature alterations, regardless of the level of peripheral virus.

After cessation of PMPA treatment, peripheral viral load recovered quickly, whereas the BSAEP latency delays reappeared 2 months after the end of treatment. Although the BSAEP abnormalities were seen 1 month after viral inoculation, the dynamics of the viral-host interaction, in the periphery as well as in the CNS, are quite different in these two conditions. Interestingly, at 1 month after inoculation, when the BSAEPs initially became abnormal, we could indeed recover virus from the CSF. In contrast, 1 month after cessation of antivi- 
ral treatment, virus could not be recovered from the $\mathrm{CSF}$, and the BSAEPs were normal; we could again begin to recover virus from the CSF of animals 2 months after treatment withdrawal, when the BSAEPs were again abnormal.

At postmortem examination, the brains of patients with HIV-related CNS symptoms frequently show parenchymal changes. Although the pathological entity of HIV encephalitis (HIVE) is purportedly correlated with HIV-induced dementia, at autopsy only approximately one half of the brains from patients with AIDS dementia showed $\operatorname{HIVE}(31,32)$. Large amounts of virus in the CNS have also been associated with functional disorders, but some patients with severe cognitive disorders have a small viral burden, whereas those without CNS disorders sometimes have far more virus (33-35). Similarly, we found a spectrum of both pathological effects and viral load in these animals. This is consistent with other findings in SIVinfected monkeys, wherein no relationship between histopathological findings and functional abnormalities could be identified (36).

Additionally, like the nonuniform distribution of HIV present in the brains of some AIDS patients at autopsy (37), we found a nonuniform spread of SIV in the brains of monkeys examined earlier in the disease course. The origin of the CNS virus at this time point is unknown and may be due to viral persistence in the CNS, rendering it not accessible to the antiviral therapy, or as a result of the increase in plasma viral load that occurred after the discontinuation of therapy, resulting in increased infection of the CNS. The heterogeneous nature of viral presence in the brain may only represent a snapshot of a dynamic process, and foci of infection and host reaction occurring in the CNS over the course of disease can contribute to an accumulation of damage over time, resulting in more severe forms of CNS dysfunction in susceptible hosts.

Interestingly, after viral inoculation, we found an acute peak elevation of MCP-1 in the CSF. MCP-1 is a potent chemoattractant not only for monocytes but also for activated $\mathrm{T}$ cells and is capable of directing the migration of monocytes, as well as some lymphocytes, across a model of the BBB (38). A gradient of chemokine concentration is required to induce chemotaxis of immune cells; thus the increased level found in the CSF relative to plasma likely contributes to an initial influx of macrophages and possibly lymphocytes into the CNS. MCP-1 is indeed elevated in the CSF of those with AIDS dementia (39-41), and an early increase in MCP1 may help seed the CNS with activated immune cells. In addition to macrophages, chemokines including MCP-1 may also contribute to the transmigration of $\mathrm{CD}^{+} \mathrm{T}$ cells (42). Importantly, we had previously reported the detection of SIV-specific cytotoxic T lymphocytes within the CSF in the first few weeks after SIV infection and in the brains of animals at sacrifice (43). The products of activated immune cells are prime candidates in mediating indirect damage to the CNS initiated by HIV infection.

A similar initial seeding of inflammatory cells and virus into the brain may contribute to CNS dysfunction in patients with HIV and may perpetuate a chronic state of low-level macrophage recruitment and immune activation in the CNS. The increased MHC class II expression that we found supports such a mechanism, and increased MHC class II expression on microglial cells has also been found in the brains of HIV-infected humans before the development of AIDS $(44,45)$. Interestingly, in a study of SIV-infected

Table 1

Histopathology and viral load in the brains of SIV-infected animals

\begin{tabular}{|c|c|c|c|c|c|c|c|c|c|c|c|c|c|c|}
\hline \multirow[t]{2}{*}{ Monkey } & \multirow[b]{2}{*}{$\begin{array}{l}\text { Inflammatory } \\
\text { cell pattern }\end{array}$} & \multicolumn{3}{|c|}{ Histopathology } & \multicolumn{2}{|c|}{ Viral load per mL } & \multicolumn{8}{|c|}{ Viral load per 25 mg tissue } \\
\hline & & $\begin{array}{l}\text { Mac387 } \\
(\mathrm{IHC})\end{array}$ & $\begin{array}{l}\text { LCA } \\
(\mathrm{IHC})\end{array}$ & $\begin{array}{c}\text { SIV } \\
\text { (in situ) }\end{array}$ & Plasma & CSF & Spleen & B:IC & $\mathrm{B}: \mathrm{CC}$ & B:CG & $\mathrm{B}: \mathrm{HC}$ & B:TG & B:HP & $\mathrm{B}: \mathrm{SC}$ \\
\hline 294 & $\begin{array}{l}\text { Mild perivascular } \\
\text { and infiltrating }\end{array}$ & ++ & ++ & + & 6.2 & 4.4 & 6.3 & $<3.2$ & $<3.2$ & $<3.2$ & $<3.2$ & $<3.2$ & $<3.2$ & $<3.2$ \\
\hline 297 & $\begin{array}{l}\text { Moderate perivascular } \\
\text { and infiltrating }\end{array}$ & +++ & ++ & + to +++ & 7.3 & 5.5 & 6.7 & 4.6 & 7.3 & 4.7 & 4.6 & 4.3 & 4.7 & 4.3 \\
\hline 299 & Mild perivascular & + & + to ++ & + & 6.5 & 3.5 & 9.4 & 4.2 & $<3.2$ & $<3.2$ & 3.8 & 3.8 & $<3.2$ & $<3.2$ \\
\hline 303 & $\begin{array}{l}\text { Perivascular and } \\
\text { focally intense infiltrating }\end{array}$ & $g^{+ \text {to }++}$ & + to +++ & 0 & $<3.2$ & $<3.2$ & 3.7 & $<3.2$ & $<3.2$ & $<3.2$ & $<3.2$ & $<3.2$ & $<3.2$ & $<3.2$ \\
\hline
\end{tabular}

The histopathology was analyzed on sections stained with hematoxylin and eosin, sections immunohistochemically (IHC) stained for activated macrophages (Mac387) and lymphocytes (LCA), and sections subjected to in situ hybridization for SIV RNA. Immune cells identified by IHC were present in a nonuniform manner, and symbols indicate the degree of cellular infiltration: 0 , no cells identified; +, one to two cells occasionally seen per high power field; ++, moderate numbers of high-power fields with more than two cells; and +++, more than four cells frequently found per high-power field. In the brains of uninfected monkeys, no Mac387-positive cells (0), and only scattered LCA-positive cells (range, 0 to +), are present. SIV-expressing cells were identified by in situ hybridization with a probe for the nef genomic region. Symbols indicate the frequency of positive cells: 0 , no cells identified; + , individual positive cells occasionally seen per tissue section; ++, moderate numbers of individual positive cells seen per tissue section; and +++, frequent individual positive cells, and clusters of positive cells, seen per tissue sections. In the brains of uninfected monkeys, no positive cells ( 0 ) are present. Viral loads are given as bDNA quantification SIV RNA copy equivalents in fluids (plasma and CSF, given as the geometric mean of the $\log _{10}$ RNA equivalents per milliliter) and tissue (spleen and brain, given as log 10 RNA equivalents per $25 \mathrm{mg}$ of tissue). The limit of detection of the assay was $3.2 \log _{10}$ RNA equivalents; samples in which the signal was not detectable are indicated by values of less than 3.2. The average of two samples is indicated. Tissue samples were obtained from the spleen and from seven regions of the brain: the inferior colliculus ( $B: I C)$, the corpus callosum (B:CC), the cingulate gyrus ( $B: C G)$, the head of the caudate (B:HC), the superior temporal gyrus ( $:$ :TG), the hippocampus (B:HP), and the striate cortex (B:SC). 
macaques, increased MHC class II expression was found in the brainstem independent of other pathology (46), and experiments in rodents revealed that microglia in the brainstem may be especially sensitive to activating stimuli (47). In addition to HIV, activation of microglia can likely lead to untoward effects on the CNS in other inflammatory or dementing diseases, including multiple sclerosis and Alzheimer's (48).

Such states of activation can contribute to the physiological changes noted here. Although in the present experiments, neither body temperature nor movement abnormalities were affected by lowering peripheral viral load, possibly peripherally produced molecules incited by a continuous host response to viral infection altered the CNS mediators of these effects. Alternatively, the sequelae of the initial viral infection and immune cell infiltration into the brain may result in a more long-standing alteration of CNS function, through mechanisms such as microglial or astroglial activation, or increased trafficking or turnover of perivascular macrophages.

Because SIV and HIV likely travel into the brain in infected cells of the immune system $(1,49)$, decreasing the peripheral viral load should reduce the probability of such infected cells from entering the CNS. Apparently such a reduction was sufficient to reverse the abnormalities in evoked potentials documented here. Similarly, reducing plasma viral load can improve at least some aspects of HIV-related neuropsychological impairment $(50,51)$. However, antiretroviral agents with better CNS penetration, or other pharmacologicals such as anti-inflammatory or neuroprotective agents, may be required to prevent the motor dysfunction and the development or persistence of untoward consequences of HIV infection on the CNS. Our findings support the assumptions that HIV infection can initiate damage to the CNS and that the CNS disorders likely result from the indirect effects of viral infection.

\section{Acknowledgments}

We thank D. Watry and M. Zandonatti for technical assistance, $M$. Buchmeier for critical reading of the manuscript, and F. Bloom for advice and support. This is manuscript no. 12521-NP from The Scripps Research Institute. This work was supported by grants from the National Institutes of Health (MH-55836, $\mathrm{MH}-59468, \mathrm{MH}-61224$, and MH-47680).

1. Price, R.W., et al. 1988. The brain in AIDS: central nervous system HIV1 infection and AIDS dementia complex. Science. 239:586-592.

2. Heaton, R.K., et al. 1994. Neuropsychological impairment in human immunodeficiency virus-infection: implications for employment. HNRC Group. HIV Neurobehavioral Research Center. Psychosom. Med. 56:8-17.

3. Michaels, S.H., Clark, R., and Kissinger, P. 1998. Declining morbidity and mortality among patients with advanced human immunodeficiency virus infection. N. Engl. J. Med. 339:405-406.

4. Moore, R.D., and Chaisson, R.E. 1999. Natural history of HIV infection in the era of combination antiretroviral therapy. AIDS. 14:1933-1942.

5. Dore, G.J., et al. 1999. Changes to AIDS dementia complex in the era of highly active antiretroviral therapy. AIDS. 13:1249-1253.

6. Schrager, L.K., and D'Souza, M.P. 1998. Cellular and anatomical reser- voirs of HIV-1 in patients receiving potent antiretroviral combination therapy. JAMA. 280:67-71.

7. Lackner, A.A. 1994. Pathology of simian immunodeficiency virus induced disease. Curr. Top. Microbiol. Immunol. 188:35-64.

8. Fox, H.S., Gold, L.H., Henriksen, S.J., and Bloom, F.E. 1997. Simian immunodeficiency virus: a model for neuroAIDS. Neurobiol. Dis. 4:265-274.

9. Zink, M.C., Spelman, J.P., Robinson, R.B., and Clements, J.E. 1998. SIV infection of macaques: modeling the progression to AIDS dementia. J. Neurovirol. 4:249-259.

10. Horn, T.F.W., Huitron-Resendiz, S., Weed, M.R., Henriksen, S.J., and Fox, H.S. 1998. Early physiological abnormalities after simian immunodeficiency virus infection. Proc. Natl. Acad. Sci. USA. 95:15072-15077.

11. Messenheimer, J.A., Robertson, K.R., Wilkins, J.W., Kalkowski, J.C., and Hall, C.D. 1992. Event-related potentials in human immunodeficiency virus infection. A prospective study. Arch. Neurol. 49:396-400.

12. Fein, G., Biggins, C.A., and MacKay, S. 1995. Delayed latency of the eventrelated brain potential P3A component in HIV disease. Progressive effects with increasing cognitive impairment. Arch. Neurol. 52:1109-1118.

13. Pagano, M.A., et al. 1992. Brain-stem auditory evoked potentials in human immunodeficiency virus-seropositive patients with and without acquired immunodeficiency syndrome. Arch. Neurol. 49:166-169.

14. Somma-Mauvais, H., and Farnarier, G. 1992. Evoked potentials in HIV infection. Neurophysiol. Clin. 22:369-384.

15. Murray, E.A., Rausch, D.M., Lendvay, J., Sharer, L.R., and Eiden, L.E. 1992. Cognitive and motor impairments associated with SIV infection in rhesus monkeys. Science. 255:1246-1249.

16. Gold, L.H., et al. 1998. Longitudinal analysis of behavioral, neurophysiological, viral and immunological effects of SIV infection in rhesus monkeys. J. Med. Primatol. 27:104-112.

17. Marcario, J.K., et al. 1999. Simple and choice reaction time performance in SIV-infected rhesus macaques. AIDS Res. Hum. Retroviruses. 15:571-583.

18. Sacktor, N.C., et al. 1996. Psychomotor slowing in HIV infection: a predictor of dementia, AIDS and death. J. Neurovirol. 2:404-410.

19. Nowak, M.A., et al. 1997. Viral dynamics of primary viremia and antiretroviral therapy in simian immunodeficiency virus infection. J. Virol. 71:7518-7525.

20. Tsai, C.C., et al. 1997. Effects of (R)-9-(2-phosphonylmethoxypropyl)adenine monotherapy on chronic SIV infection in macaques. AIDS Res. Hum. Retroviruses. 13:707-712.

21. Dailey, P.J., Zamround, M., Kelso, R., Kolberg, J., and Urdea, M. 1995. Quantitation of simian immunodeficiency virus (SIV) RNA in plasma of acute and chronically infected macaques using a branched DNA (bDNA) signal amplification assay. J. Med. Primatol. 24:209. (Abstr.)

22. Terrault, N.A., et al. 1997. Hepatitis C virus: quantitation and distribution in liver. J. Med. Virol. 51:217-224.

23. Connor, R.I., et al. 1998. Temporal analyses of virus replication, immune responses, and efficacy in rhesus macaques immunized with a live, attenuated simian immunodeficiency virus vaccine. J. Virol. 72:7501-7509.

24. Shaw, J.P., et al. 1997. Metabolism and pharmacokinetics of novel oral prodrugs of 9-[(R)-2-(phosphonomethoxy)propyl]adenine (PMPA) in dogs. Pharm. Res. 14:1824-1829.

25. Lane, T.E., Buchmeier, M.J., Watry, D.D., and Fox, H.S. 1996. Expression of inflammatory cytokines and inducible nitric oxide synthase in brains of SIV-infected rhesus monkeys: applications to HIV-induced central nervous system disease. Mol. Med. 2:27-37.

26. Prospero-Garcia, O., et al. 1996. Microglia-passaged simian immunodeficiency virus induces neurophysiological abnormalities in monkeys. Proc. Natl. Acad. Sci. USA. 93:14158-14163.

27. Weed, M.R., et al. 1999. Performance norms for a rhesus monkey neuropsychological testing battery: acquisition and long-term performance. Brain Res. Cogn. Brain Res. 8:185-201.

28. Smith, M.O., Sutjipto, S., and Lackner, A.A. 1994. Intrathecal synthesis of IgG in simian immunodeficiency virus (SIV)-infected rhesus macaques (Macaca mulatta). AIDS Res. Hum. Retroviruses. 10:81-89.

29. Balzarini, J., Sobis, H., Naesens, L., Vandeputte, M., and De Clercq, E. 1990. Inhibitory effects of 9-(2-phosphonylmethoxyethyl)adenine and 3'-azido-2',3'-dideoxythymidine on tumor development in mice inoculated intracerebrally with Moloney murine sarcoma virus. Int. J. Cancer. 45:486-489

30. Lane, J.H., et al. 1996. Neuroinvasion by simian immunodeficiency virus coincides with increased numbers of perivascular macrophages/microglia and intrathecal immune activation. J. Neurovirol. 2:423-432.

31. Navia, B.A., Cho, E.S., Petito, C.K., and Price, R.W. 1986. The AIDS dementia complex. II. Neuropathology. Ann. Neurol. 19:525-535.

32. Glass, J.D., Wesselingh, S.L., Selnes, O.A., and McArthur, J.C. 1993. Clinical-neuropathologic correlation in HIV-associated dementia. Neurology. 43:2230-2237.

33. Wiley, C.A., et al. 1991. Neocortical damage during HIV infection. Ann. Neurol. 29:651-657.

34. Brew, B.J., Rosenblum, M., Cronin, K., and Price, R.W. 1995. AIDS 
dementia complex and HIV-1 brain infection: clinical-virological correlations. Ann. Neurol. 38:563-570.

35. Masliah, E., et al. 1997. Dendritic injury is a pathological substrate for human immunodeficiency virus-related cognitive disorders. HNRC Group. The HIV Neurobehavioral Research Center. Ann. Neurol. 42:963-972.

36. Rausch, D.M., Murray, E.A., and Eiden, L.E. 1999. The SIV-infected rhesus monkey model for HIV-associated dementia and implications for neurological diseases. J. Leukoc. Biol. 65:466-474.

37. Wiley, C.A., et al. 1998. Distribution of brain HIV load in AIDS. Brain Pathol. 8:277-284.

38. Weiss, J.M., Downie, S.A., Lyman, W.D., and Berman, J.W. 1998. Astrocyte-derived monocyte-chemoattractant protein-1 directs the transmigration of leukocytes across a model of the human blood-brain barrier. J. Immunol. 161:6896-6903.

39. Conant, K., et al. 1998. Induction of monocyte chemoattractant protein1 in HIV-1 Tat-stimulated astrocytes and elevation in AIDS dementia. Proc. Natl. Acad. Sci. USA. 95:3117-3121.

40. Cinque, P., et al. 1998. Elevated cerebrospinal fluid levels of monocyte chemotactic protein-1 correlate with HIV-1 encephalitis and local viral replication. AIDS. 12:1327-1332.

41. Kelder, W., McArthur, J.C., Nance-Sproson, T., McClernon, D., and Griffin, D.E. 1998. Beta-chemokines MCP-1 and RANTES are selectively increased in cerebrospinal fluid of patients with human immunodeficiency virus-associated dementia. Ann. Neurol. 44:831-835.

42. Rabin, R.L., et al. 1999. Chemokine receptor responses on T cells are achieved through regulation of both receptor expression and signaling. J. Immunol. 162:3840-3850.

43. von Herrath, M., Oldstone, M.B., and Fox, H.S. 1995. Simian immunodeficiency virus (SIV)-specific CTL in cerebrospinal fluid and brains of SIV-infected rhesus macaques. J. Immunol. 154:5582-5589.

44. An, S.F., et al. 1996. Investigation on the expression of major histocompatibility complex class II and cytokines and detection of HIV-1 DNA within brains of asymptomatic and symptomatic HIV-1-positive patients. Acta Neuropathol. (Berl). 91:494-503.

45. Gray, F., et al. 1996. Neuropathology of early HIV-1 infection. Brain Pathol. 6:1-15

46. Berman, N.E.J., et al. 1998. Gradient of microglial activation in the brain of SIV infected macaques. J. Neuro-AIDS. 2:43-54.

47. Phillips, L.M., Simon, P.J., and Lampson, L.A. 1999. Site-specific immune regulation in the brain: differential modulation of major histocompatibility complex (MHC) proteins in brainstem vs. hippocampus. J. Comp. Neurol. 405:322-333.

48. Gonzalez-Scarano, F., and Baltuch, G. 1999. Microglia as mediators of nflammatory and degenerative diseases. Annu. Rev. Neurosci. 22:219-240.

49. Haase, A.T. 1986. Pathogenesis of lentivirus infections. Nature. 322:130-136.

50. Ferrando, S., et al. 1998. Highly active antiretroviral treatment in HIV infection: benefits for neuropsychological function. AIDS. 12:F65-F70.

51. Sacktor, N.C., et al. 1999. Combination antiretroviral therapy improves psychomotor speed performance in HIV-seropositive homosexual men. Multicenter AIDS Cohort Study (MACS). Neurology. 52:1640-1647. 